Aims This mixed-methods study aimed to (i) quantify the extent and risk of bias of MD in palliative care trials; (ii) explore the factors associated with MD, (iii) develop MD recommendations for patients, clinicians, researchers and policymakers.

Methods (i) Systematic review of MD in 108 palliative care trials (meta-analyses and meta-regression); (ii) individual participant-level data (IPD) meta-analysis of factors associated with MD in 10 palliative care trials (multi-level modelling); (iii) indepth interviews with research personnel (thematic analysis).

Results (i) Systematic review: 23\% of primary outcome data were missing at the primary end-point (95\% CI 19, 27). Larger MD proportions were associated with increasing numbers of questions/tests asked of participants (odds ratio (OR) 1.2 per-doubling, 95\% CI 1.1, 1.4) and longer study duration (OR 1.1 per-doubling, 95\% CI 1.0, 1.2). There was evidence of differential rates of MD between trial arms, thus potentially introducing bias. (ii) IPD: Participants with previous MD (OR 17.2, 95\% CI 8.6, 34.5) and poorer Karnofsky Performance Status (10-unit increase: OR 0.8, 95\% CI 0.7, 0.9) were more likely to have MD. (iii) Interviews: themes included 'clinical vs research-role tension' and 'attention-to-detail vs attentionto-person'.

Conclusions MD pose a significant risk to palliative care trial findings but can potentially be reduced. It is therefore essential that multi-disciplinary team members can (i) critically assess the MD risk of bias and (ii) help to reduce MD - guidance on how to do these will be discussed.

\section{0-7 HOW SYSTEM-WIDE ADVANCE CARE PLANNING SUPPORT CAN BE DELIVERED COST EFFECTIVELY}

Josie Dixon, Martin Knapp. London School of Economics and Political Science, London, UK

\subsection{6/bmjspcare-2018-hospiceabs.7}

Advance care planning (ACP) can promote person-centred care and better use of resources. ACP involving a facilitated conversation with a health or care professional is more effective than document completion alone. However, these conversations are time consuming. Evidence about how ACP support can be delivered by health and care professionals cost-effectively and at scale is currently lacking.

To explore this issue, we identified twelve international healthcare organisations with practical experience of resourcing, staffing and delivering system-wide ACP support. In each, we conducted an average 13 in-depth interviews with senior managers, ACP leads, dedicated ACP facilitators, physicians, nurses, social workers and other clinical and non-clinical staff. Data were thematically analysed using NVivo software.

Our findings emphasised the importance of strategic and dedicated day-to-day leadership for creating accountability and supporting professionals to deliver ACP support within their roles. Reliance on dedicated facilitators was not considered sustainable or scalable, although a degree of staff specialism improved efficiency and effectiveness and allowed the better targeting of training.

A lack of protected time was commonly experienced as a barrier. Incorporating ACP into existing care could mean it got 'squeezed out' or became too fragmented. Physician leadership was important, but entirely physician-led approaches were associated with late introduction and limited scope. Team-based approaches, involving nurses and social workers, were recommended for cost-efficiency and quality of care, and were well-aligned well with new models of care.

When used by experienced staff, the choice of ACP conversation tool had limited influence on the length of a conversation. Participants' informational and educational needs, however, significantly influenced the time required for a conversation. There was scope for efficiencies here from the use of decision aids, educational events and activities in the community and group-based facilitations in community and clinical settings.

\section{Parallel session 3: No child left behind? The challenges we face in caring for children}

\section{0-8 NURSES' PERCEPTIONS OF CARING FOR CHILDREN AFTER ORGAN DONATION IN CHILDREN'S HOSPICE COOL ROOMS}

${ }^{1}$ Michael Tatterton, ${ }^{1}$ Caroline Brennan, ${ }^{2}$ Rachel Summers. 'Martin House Children's Hospice, Wetherby, UK; ${ }^{2}$ Yorkshire Organ Donation Services Team, NHS Blood and Transplant, Leeds, UK

\subsection{6/bmjspcare-2018-hospiceabs.8}

Background In Yorkshire, there were five paediatric organ donors in 2017/18. Very limited bereavement support is offered to families whose children donate organs (Berntzen \& Bjørk, 2014). Changes to the Specialist Nurse Organ Donation (SNOD) role meant that SNODs felt unable to provide donor families with adequate bereavement support. This led to a broadening of hospice acceptance criteria, where deceased children, without known life-limiting conditions (Widdas, $\mathrm{McNa}$ mara \& Edwards, 2013) could use the cool bedrooms (Forrester, 2008) for post-mortem care, and their families access bereavement support offered by the hospice. Since $2017 / 18$, a total of four children have been cared for postdonation.

Aim To explore the perceptions and experience of registered nurses involved in caring for families of children and young people who have donated organs in children's hospice cool rooms.

Methods A semi-structured focus group was conducted with nurses from the Yorkshire Organ Donation Services Team, and Martin House in March 2018. Purposeful sampling was used (Richie, Lewis, McNaughton Nicholls et al., 2013), based on nurses who either:

- Whilst working as a SNOD, referred a child and family to the hospice following organ donation

- Whilst working at the hospice, cared for the body of a child after organ donation, and their family.

Results Five core themes emerged from the thematic analysis: (i) barriers that prevented families from receiving the care they need, (ii) bereavement support, (iii) impact: on families, SNODs and professionals, (iv) influencers and enablers of change, and (v) sustainability of services.

Conclusions Nurses perceived that families gained much from the bereavement support offered by the hospice, and from being able to spend time with their child's body after death. Although some of the grief experiences of families was different to what hospice nurses were used to, staff gained confidence as their exposure increased. Joint working between the 